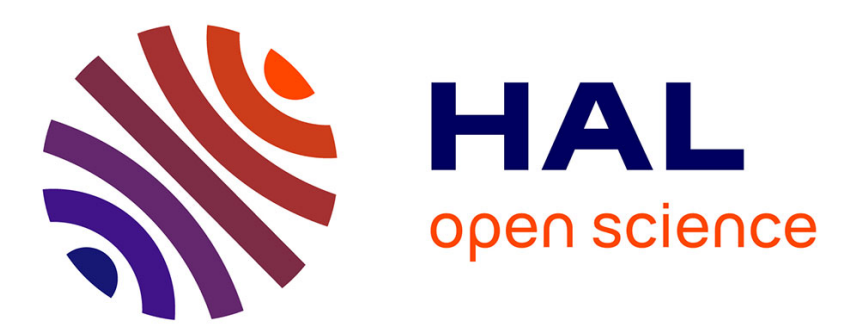

\title{
Spatially regularized wavelet leader scale-free analysis of fMRI data
}

\author{
Herwig Wendt, Patrice Abry, Philippe Ciuciu
}

\section{To cite this version:}

Herwig Wendt, Patrice Abry, Philippe Ciuciu. Spatially regularized wavelet leader scale-free analysis of fMRI data. IEEE International Symposium on Biomedical Imaging, Apr 2018, Washington, DC, United States. hal-01782332

\section{HAL Id: hal-01782332 \\ https://hal.inria.fr/hal-01782332}

Submitted on 1 May 2018

HAL is a multi-disciplinary open access archive for the deposit and dissemination of scientific research documents, whether they are published or not. The documents may come from teaching and research institutions in France or abroad, or from public or private research centers.
L'archive ouverte pluridisciplinaire HAL, est destinée au dépôt et à la diffusion de documents scientifiques de niveau recherche, publiés ou non, émanant des établissements d'enseignement et de recherche français ou étrangers, des laboratoires publics ou privés. 


\title{
SPATIALLY REGULARIZED WAVELET LEADER SCALE-FREE ANALYSIS OF FMRI DATA
}

\author{
Herwig Wendt ${ }^{1}$, Patrice Abry ${ }^{2}$ and Philippe Ciuciu ${ }^{3}$ \\ (1) IRIT-ENSHEEIT, CNRS, Univ. Toulouse, France, herwig.wendteirit.fr \\ (2) Univ Lyon, Ens de Lyon, Univ Claude Bernard, CNRS, Laboratoire de Physique, Lyon, France, \\ patrice.abrydens-lyon.fr \\ (3) CEA/NeuroSpin and INRIA-CEA Parietal, Univ. Paris-Saclay, France, philippe.ciuciu@cea.fr
}

\begin{abstract}
Slow brain dynamics has received considerable interest in the recent years, with the scale-free paradigm playing a crucial role for analysis of various neuroimaging modalities. However, assessing the role of slow arrhythmic fluctuations requires the use of a large continuum of time scales and thus of long time series, hence raising concerns regarding the use of scale-free tools on fMRI data. Further, scale-free analysis remained so far mostly univariate, that is, voxels are analyzed independently, hence neglecting their spatial organization. The present contribution aims to propose a spatially regularized estimation of the self-similarity parameter, based on a recently formalized formalism combining wavelet leaders and Bayesian models. The spatially regularized estimates permit to quantify the modulations of the scale-free dynamics from rest to a working memory task from fMRI data collected for 21 healthy volunteers. These modulations are significant in the default mode network and in some regions involved in task performance such as primary visual regions or the supplementary motor area.
\end{abstract}

Index Terms - fMRI, scale-free, multivariate, spatial regularization, Bayesian inference.

\section{INTRODUCTION}

Scale-free dynamics in macroscopic brain activity. Energy levels and temporal dynamics of oscillations in pre-defined frequency bands, that quantify the behaviors of populations of neurons, remain reference tools to model and analyze macroscopic brain activity, assessed by M/EEG measurements. However, slow activities $(<2 \mathrm{~Hz})$ are a prominent part of functional brain dynamics. They are nowadays often modeled using the scale-free paradigm, postulating arrhythmic (oscillation free) temporal dynamics. Due to hemodynamic filtering, these arrhythmic dynamics lie below $0.2 \mathrm{~Hz}$ in fMRI data. Indeed, scale-free temporal dynamics were observed across several modalities, both at rest or during

Work supported by ANR-16-CE33-0020 MultiFracs, France task performance, and under various conditions or pathologies [1-12]. It has also been documented that scale-free dynamics are functionally associated with neural excitability $[3,13]$ and negatively correlates with power fluctuation in alpha-band $(8-12 \mathrm{~Hz})$ [14], hence explaining the modulation of scaling exponents with task engagement or pathologies.

Related works. It is well-established that scale-free temporal dynamics are well modeled by self-similar processes, such as fractional Brownian motion. Wavelet tranforms permit a theoretically well-grounded and practically robust assessment of scale-free dynamics and estimation of the corresponding scaling or Hurst exponent (cf. e.g., [15] for the methodology and $[9,10]$ for applications to fMRI data). More recently, it has however been shown that the use of wavelet leaders, a non-linear non-local transformation of wavelet coefficients, permits to better account for the richness and variety of scalefree dynamics actually observed in data, and yields estimation procedures for $H$ with better performance (used here, cf. Section 2), cf. e.g., [16] for the methodology and [9] for applications in neurociences. Since scale-free analysis requires the data to be analyzed at several time scales jointly, relatively long time series are required for robust estimation. This limitation may explain why it leads to relevant characterizations and promising conclusions when applied to modalities such as M/EEG $[6,11,12]$ while successes remain debated on (typically short length) fMRI time series. Another limitation stems from current scale-free analysis procedures being univariate: Despite several tens of thousands of voxels being recorded jointly in the brain with fMRI, the obviously rich and informative spatial structure of the data is not exploited and each time series is analyzed independently.

Goals, contributions and outline. The present contribution aims to overcome the limitations caused by the small sample size of fMRI time series by taking advantage of their multivariate nature. Preliminary attempt to cope with spatial regularization on fMRI data for the estimation of $H$ has been conducted in [17] in a variational framework. Here, elaborating on the general methodology proposed in $[18,19]$, a Bayesian spatial regularization procedure for the estimation of the selfsimilarity parameter $H$, exploiting the statistical properties of 
wavelet leaders, is devised in Section 2. This spatially regularized estimation procedure is illustrated on fMRI data, collected on a cohort of 21 healthy volunteers, both at rest and while performing a working memory task (cf. Section 3 for details about the experiment). Section 4 shows the benefits and potential of this novel multivariate estimation procedure to quantify the modulations of scale-free dynamics from rest to task on the whole brain, as compared to univariate scalefree analysis.

\section{BAYESIAN SPATIALLY REGULARIZED SCALE-FREE ANALYSIS}

\subsection{Univariate procedures}

Wavelet leaders. For a time series $X$, discrete wavelet transform coefficients $d_{X}(j, k)$ are computed as $d_{X}(j, k)=$ $\left\langle X, 2^{-j / 2} \psi_{j, k}\right\rangle$, where the collection $\left\{\psi_{j, k}(t) \equiv 2^{-j / 2} \psi_{0}\left(2^{-j} t-\right.\right.$ $k), j \in \mathbb{N}, k \in \mathbb{N}\}$ is constructed by dilations and translations of a the mother-wavelet $\psi_{0}(t)$. It is characterized by its number of vanishing moments $N_{\psi} \geq 1\left(\forall k=0, \ldots, N_{\psi}-1\right.$, $\int_{\mathbb{R}} t^{k} \psi_{0}(t) d t \equiv 0$ and $\left.\int_{\mathbb{R}} t^{N_{\psi}} \psi_{0}(t) d t \neq 0\right)$.

Wavelet leaders are further constructed as local suprema of wavelet coefficients, taken over finer scales and within a short temporal neighborhood $3 \lambda_{j, k}$, with $\lambda_{j, k}=\left[k 2^{j},(k+\right.$ 1) $2^{j}$ ) the dyadic interval of size $2^{j}$ and $3 \lambda_{j, k}$ the union of $\lambda_{j, k}$ with its 2 neighbors [16]: $L(j, k)=\sup _{\lambda^{\prime} \subset 3 \lambda_{j, k}}\left|d_{X}\left(\lambda^{\prime}\right)\right|$.

Linear regression. A relevant wavelet leader based estimator for the self-similarity parameter $H$ can be constructed on the linear regression of the averaged $\log$ leaders, $\ell(j, k):=$ $\ln L(j, k)$, across scales ( with weights $w_{j}$ selected to produce an ordinary least square estimate), cf. [16]:

$$
\hat{H}_{L F}=\frac{1}{\ln 2} \sum_{j=j_{1}}^{j_{2}} w_{j} \frac{1}{n_{j}} \sum_{k=1}^{n_{j}} \ell(j, k) .
$$

Bayesian estimation. A wavelet leader Bayesian estimator for $H$ can be further developed. It exploits the approximative modeling of the statistical properties of $\ell(j, k)$ as multivariate normal distributions as proposed in $[18,19]$ :

$$
\left[\ell(j, 1), \ldots, \ell\left(j, n_{j}\right)\right] \sim \mathcal{N}\left(c+H \ln \left(2^{j}\right), \Sigma_{j, z}\right),
$$

with $\Sigma_{j, z}$ a semiparametric model for the covariance at scale $2^{j}[18,19]$. Parameter $z$ controlling the variance is estimated using Bayesian scheme and priors as detailed in $[18,19]$. The use of Gaussian priors for the parameter vectors $(c, H)$ leads to a Gaussian posterior distribution and permits straightforward computation of the marginal posterior mean estimator for $H$ defined as

$$
\hat{H}_{B U}=\mathbb{E}[H \mid \ell] .
$$

When non-informative Gaussian priors are used, $\hat{H}_{B U}$ in (3) takes a form similar to $\hat{H}_{L F}$ in (1), yet with weights $w_{j}$ that are estimated as an output of the Bayesian scheme and reflect the variances of $\ell(j, k)$, at each scale $2^{j}$.

\subsection{Bayesian spatially regularized procedures}

With the procedure described above, the estimates $\hat{H}_{v}$ for all voxels $v$ are performed independently one from another. To take advantage of the spatial organization of the voxels and to improve the estimation of the collection of parameters $\underline{\mathrm{H}}=\left\{H_{v}\right\}_{v=1}^{n_{v o x e l}}$, the statistical model leading to (3) can be extended to a multivariate setting and embedded into a hierarchical Bayesian model incorporating spatial regularization. Instead of Gaussian priors that are independent for each voxel in $\hat{H}_{B U}$, use is made of spatially regularizing joint priors to favor smooth variations of parameter values between adjacent voxels. They penalize second order spatial differences $D \underline{H}$, with $D$ the 3D discrete Laplacian operator [20]:

$$
p(\underline{\mathbf{H}}, \epsilon) \propto \epsilon^{-\frac{1}{2} \operatorname{rank}\left(D^{T} D\right)} \exp \left(-\frac{1}{2 \epsilon}\|D \underline{\mathbf{H}}\|^{2}\right) p(\epsilon) .
$$

The hyperprior $p(\epsilon)$ controls the amount of spatial regularization. With the log-leaders of each voxel being modeled as independent with likelihood (2), this prior leads to closed from Gaussian and inverse gamma conditional distributions for $\underline{\mathrm{H}}$ and $\epsilon$, respectively. This hence permits the efficient approximation of the marginal posterior mean estimator used to define the spatially regularized multivariate estimator for $\underline{\mathrm{H}}$ :

$$
\underline{\hat{\mathrm{H}}}_{B M}=\mathbb{E}\left[\underline{\mathrm{H}} \mid\left\{\ell_{v}\right\}_{v=1}^{n_{v o x e l}}\right] .
$$

\section{EXPERIMENT DESIGN AND FMRI DATA}

Working Memory Task. A group of 21 (female) participants were subjected to a verbal working memory task. Subjects had to attend visual sequences of serially presented uppercase letters, spanning the entire alphabet and displayed during $1 \mathrm{~s}$ with an inter-stimuls interval of $2 \mathrm{~s}$. The working memory paradigm included a sequence of 8 blocks comprising 0-back and 2-back tasks. In the control (0-back) task, subjects had to identify the occurrence of the letter $X$. In the 2-back task, subjects had to determine whether each presented letter was the same as that presented 2 stimuli before. The ratio of targets to distractors was about $30 \%$ within each block. Participants were instructed to respond to targets by pushing a button with their right thumb. Responses and reaction times were recorded.

Data collection. fMRI recordings were achieved using a 3 Tesla on a Siemens Trio system (Erlangen, Germany), both at rest and during working memory tasks. During resting-state, participants were instructed to keep eyes closed. A multiband GE-EPI $\left(\mathrm{TE}=30 \mathrm{~ms}, \mathrm{TR}=1 \mathrm{~s}, \mathrm{FA}=61^{\circ}, \mathrm{mb}=2\right)$ sequence (CMRR, Minneapolis, USA) was used, with 3-mm isotropic resolution and a FOV of $192 \times 192 \times 144 \mathrm{~mm}^{3} .543$ scans were collected for a total recording time of 9 min10s. Task-related fMRI data were collected using the same experimental setup except that only 512 images $(8 \mathrm{~min} 39 \mathrm{~s})$ were acquired. 

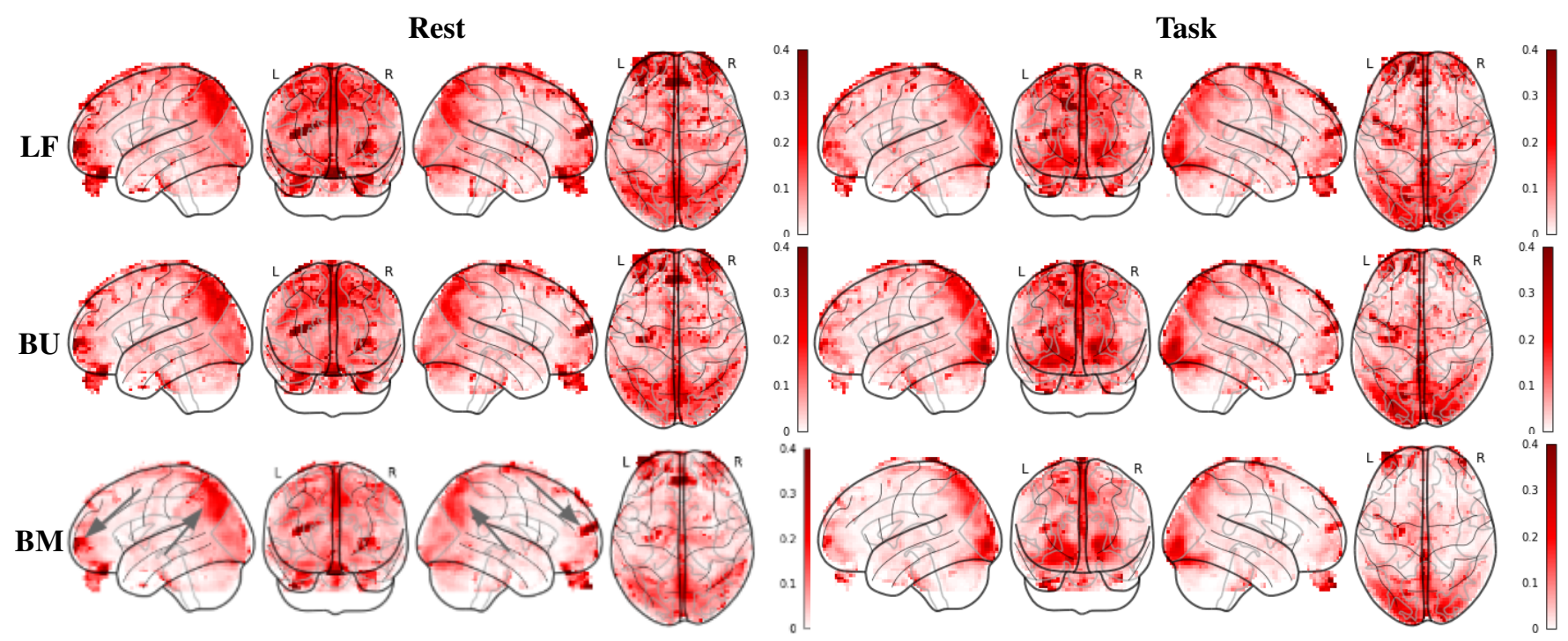

Fig. 1. Group-level estimates of $H$. From top to bottom $\hat{H}_{L F}-1, \hat{H}_{B U}-1$ and $\hat{H}_{B M}-1$ (1 has been substratced to enhance contrast). From left to right, left-sagittal, coronal, right-sagittal, axial brain slices. These figures have been designed using http://nilearn.github.io. Gray-colored arrows indicate brain regions (PCC, FP) where the multivariate estimate $\hat{H}_{B M}$ gives enhanced contrast.

\section{RESULTS AND DISCUSSION}

Analysis setting. Wavelet analysis was performed using a Daubechies least asymmetric wavelets, with $N_{\psi}=3$ vanishing moments. Scales $2^{2} \leq 2^{j} \leq 2^{5}$ were used for the estimation of the Hurst parameters, essentially corresponding to frequencies in the range $0.02 \leq f \leq 0.2$.

Comparing the different estimates $\hat{H}$. Fig. 1 reports the group-level whole brain estimates of $H$ across different brain views, for the three different estimation procedures $\hat{H}_{L F}, \hat{H}_{B U}$ and $\hat{H}_{B M}$, independently for rest and task. As explained in Section $2, \hat{H}_{L F}$ and $\hat{H}_{B U}$ procedures yield similar maps of estimates. In contrast, a qualitative comparison of $\hat{H}_{B M}$ against $\hat{H}_{L F}$ or $\hat{H}_{B U}$ shows a better contrasted map of estimates, that can be interpreted as a denoising of $\hat{H}_{B U}$. At rest, $\hat{H}_{B M}$ preserves large structures with homogeneous estimates of $H$, such as for the Posterior Cingulate Cortex (PCC), a central part of the Default Mode Network (DMN), and, in addition enhances contrasts and better localizes smaller size regions, such as the Fronto-Polar region, another DMN component. Further, $\hat{H}_{B M}$ for the frontal lobe shows homogeneously low estimates of $H$ compared to spurious localized fluctuations observed for $\hat{H}_{L F}$ and $\hat{H}_{B U}$. During task, the same denoising and enhancing effects are qualitatively visible.

These qualitative analyses illustrate the benefits of the spatially regularized estimation procedures, compared to the univariate ones. Interestingly, $\hat{H}_{B M}$ permits to show that, at rest, the largest values of $H$ are observed mainly in the DMN, thus confirming its central role in brain functioning at rest, in agreement with results reported on region-based analysis in the literature, cf. [5]. Also, during task, large $H$ are observed in the Primary Visual Cortex, involved in the task, as it elicits evoked activity in response to visual stimuli.

Rest versus Task. To statistically assess differences between $H$ at rest and during task, Fig. 2 reports the superimposition of the two one-sided paired t-tests rejecting the null hypothesis $\hat{H}^{(r)} \equiv \hat{H}^{(t)}$ (thresholded at $|T|>4$, i.e., uncorrected p-value of $3.5 \cdot 10^{-4}$ ). It shows that, from rest to task, $H$ significantly decreases in several parts of the DMN (red spots in Fig. 2), indicating a decrease of the long range correlation. This is notably the case for the Bilateral Angular Gyri, Brodmann Area (BA) 39, and Bilateral Precuneus, part of PCC and functional core of DMN. This decrease in $H$ can be interpreted as the active inhibition (or deactivation) of DMN during task performance, in agreement with [5]. Interestingly, this decrease results in a clean segmentation of parts of the DMN, obtained without computing seed-based functional connectivity with seed located a priori in PCC.

It also shows that in other regions $H$ significantly increases from rest to task (blue zones in Fig. 2), thus implying a strengthening of the correlation structure. This is notably the case for Medial PreFrontal Cortex (including Supplementary Motor Area (BA 6)) which is involved in the planification for motor response (button press required by $n$-back task). This change in temporal dynamics is consistent with the taskrelated activity reported in the meta-analysis of $n$-back fMRI protocols in [21].

It is also worth mentioning that substantial changes in $H$, hence in temporal dynamics, are not seen across the whole fronto-parietal working memory network region involved in $n$-back task. 


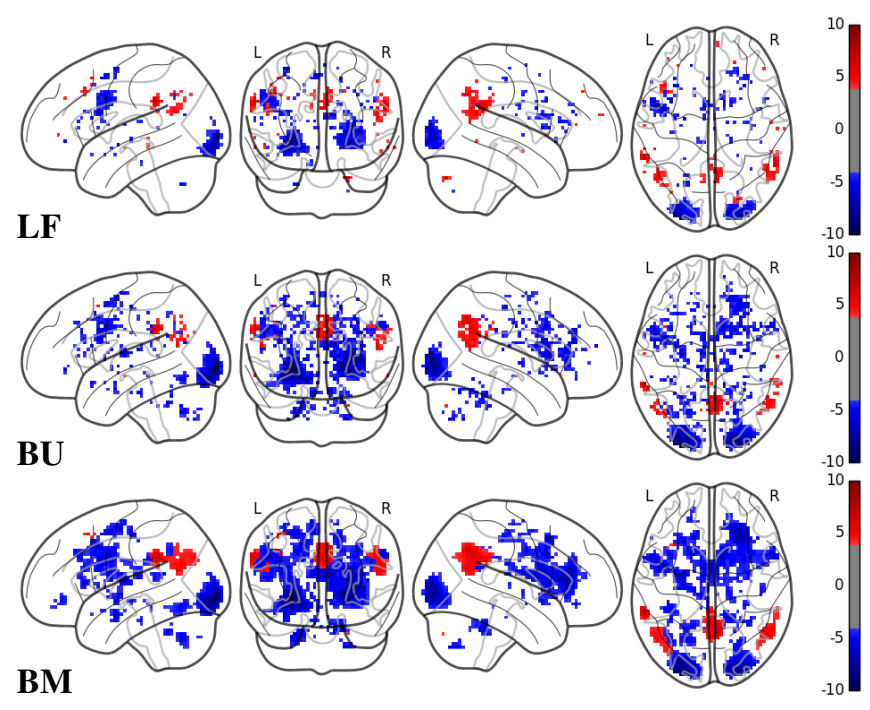

Fig. 2. T-test for rejecting $\hat{H}^{(r)}=\hat{H}^{(t)}$. From top to bottom $\hat{H}_{L F}^{(r)}-\hat{H}_{L F}^{(t)}, \hat{H}_{B U}^{(r)}-\hat{H}_{B U}^{(t)}$ and $\hat{H}_{B M}^{(r)}-\hat{H}_{B M}^{(T)}$. Red: $\hat{H}^{(r)}>$ $\hat{H}^{(t)}$, Blue: $\hat{H}^{(r)}<\hat{H}^{(t)}$. From left to right, left-sagittal, coronal, right-sagittal, axial brain slices.

\section{CONCLUSION AND PERSPECTIVES}

The benefits and potential of the wavelet leader based spatially regularized Bayesian estimation for scale-free parameters has been well evidenced. It indicates that the limitation stemming from the low sample-size of fMRI time series can be efficiently overcome by making use of their spatial structure. This will be complemented by extending the spatial regularization to the analysis of multifractal properties in fMRI data, which enable to generalize the description of scale-free temporal dynamics beyond the sole self-similarity parameter $H$ to higher order dependence structure $[18,19,22]$. This work will be continued with the study of a larger number of subjects and of the impact of prescribed medication on the modulation of scale-free temporal dynamics.

\section{REFERENCES}

[1] A Eke, P Herman, L Kocsis, and LR Kozak, "Fractal characterization of complexity in temporal physiological signals," Physiological measurement, vol. 23, no. 1, pp. R1, 2002.

[2] Ed Bullmore, Jalal Fadili, Voichita Maxim, Levent Şendur, Brandon Whitcher, John Suckling, Michael Brammer, and Michael Breakspear, "Wavelets and functional magnetic resonance imaging of the human brain," Neuroimage, vol. 23, pp. S234-S249, 2004.

[3] V. Maxim, L. Sendur, J. Fadili, J. Suckling, R. Gould, R. Howard, and E. Bullmore, "Fractional Gaussian noise, functional MRI and Alzheimer's disease," Neuroimage, vol. 25, no. 1, pp. 141-158, 2005.

[4] B. J. He, J. M. Zempel, A. Z. Snyder, and M. E. Raichle, "The temporal structures and functional significance of scale-free brain activity," Neuron, vol. 66, no. 3, pp. 353-369, 2010.

[5] B. J. He, "Scale-free properties of the functional magnetic resonance imaging signal during rest and task," J. Neurosci., vol. 31, no. 39, pp. 13786-13795, Sep. 2011.
[6] D. Van de Ville, J. Britz, and Ch. Michel, "EEG microstate sequences in healthy humans at rest reveal scale-free dynamics," Proc. Nat. Acad. Sci. (PNAS), vol. 107, no. 42, pp. 18179-84, 2010.

[7] N. Dehghani, C. Bedard, S. S. Cash, E. Halgren, and A. Destexhe, "Comparative power spectral analysis of simultaneous elecroencephalographic and magnetoencephalographic recordings in humans suggests non-resistive extracellular media," J. Comput. Neurosci., vol. 29, no. 3, pp. 405-421, 2010.

[8] P. Ciuciu, P. Abry, C. Rabrait, and H. Wendt, "Log wavelet leaders cumulant based multifractal analysis of EVI fMRI time series: evidence of scaling in ongoing and evoked brain activity," IEEE J. Selected Topics in Signal Process., vol. 2, no. 6, pp. 929-943, 2008.

[9] P. Ciuciu, G. Varoquaux, P. Abry, S. Sadaghiani, and A. Kleinschmidt, "Scale-free and multifractal time dynamics of fMRI signals during rest and task," Front. Physiol., vol. 3, June 2012.

[10] P. Ciuciu, P. Abry, and B. J. He, "Interplay between functional connectivity and scale-free dynamics in intrinsic fMRI networks," Neuroimage, vol. 95, pp. 248-263, 2014.

[11] N. Zilber, P. Ciuciu, P. Abry, and V. van Wassenhove, "Modulation of scale-free properties of brain activity in MEG," in Proc. of the 9th IEEE Int. Symp. Biomed. Imaging (ISBI), Barcelona, Spain, 2012, pp. $1531-1534$.

[12] K. Gadhoumi, J. Gotman, and J.-M. Lina, "Scale invariance properties of intracerebral eeg improve seizure prediction in mesial temporal lobe epilepsy," PloS one, vol. 10, no. 4, 2015.

[13] B. J. He, "Scale-free brain activity: past, present, and future," Trends in Cognitive Sci., vol. 18, no. 9, pp. 480-487, 2014.

[14] Robert Becker, Dimitri Van De Ville, and Andreas Kleinschmidt, "Alpha oscillations reduce temporal long-range dependence in spontaneous human brain activity," Journal of Neuroscience, 2017.

[15] D. Veitch and P. Abry, "A wavelet based joint estimator of the parameters of long-range dependence," IEEE T. Info. Theory, vol. 45, no. 3, pp. 878-897, 1999.

[16] H. Wendt, P. Abry, and S. Jaffard, "Bootstrap for empirical multifractal analysis," IEEE Signal Proc. Mag., vol. 24, no. 4, pp. 38-48, 2007.

[17] H. Pellé, P. Ciuciu, M. Rahim, E. Dohmatob, P. Abry, and V. van Wassenhove, "Multivariate Hurst exponent estimation in fMRI. Application to brain decoding of perceptual learning," in 13th Proc. IEEE ISBI, Prague, Czech Republic, Apr. 2016, pp. 996-1000.

[18] S. Combrexelle, H. Wendt, N. Dobigeon, J.-Y. Tourneret, S. McLaughlin, and P. Abry, "Bayesian estimation of the multifractality parameter for image texture using a Whittle approximation," IEEE T. Image Proces., vol. 24, no. 8, pp. 2540-2551, 2015.

[19] S. Combrexelle, H. Wendt, Y. Altmann, J.-Y. Tourneret, S. McLaughlin, and P. Abry, "Bayesian estimation for the local assessment of the multifractality parameter of multivariate time series," in Proc. EUSIPCO, Budapest, Hungary, September 2016.

[20] N. Cressie, Statistics for spatial data, John Wiley \& Sons, 2015.

[21] A. M. Owen, K. M. McMillan, A. R. Laird, and E. Bullmore, "N-back working memory paradigm: A meta-analysis of normative functional neuroimaging studies," Human Brain Mapping, vol. 25, no. 1, pp. 46$59,2005$.

[22] P. Ciuciu, H. Wendt, S. Combrexelle, and P. Abry, "Spatially regularized multifractal analysis for fMRI data," in International IEEE EMBS Conference (EMBC), Jeju, South Korea, July 2017. 